\title{
Subsunción del conocimiento científico al capital Reflexión desde el desarrollo de las nanotecnologías
}

\author{
Domingo Rafael Castañeda Olvera* \\ Edgar Záyago Lau**
}

Resumen. La dinámica histórica de acumulación que ha logrado integrar a las economías mundiales ha viabilizado la subsunción de la ciencia y la tecnología al capital; las nanotecnologías no escapan a esta realidad. El conocimiento se ha convertido en un espacio de interés para la revalorización, y sus secuelas pueden implicar un incremento de las desigualdades sociales en el orbe. En este trabajo se analizan las implicaciones de tal subsunción y cómo se manifiesta en las nanotecnologías. El objetivo es promover la reflexión, desde una visión crítica, sobre el desarrollo de dicho paquete tecnológico revolucionario.

Palabras clave: subsunción de la ciencia, conocimiento, nanotecnologías, investigación, desarrollo.

*Profesor investigador en la División Académica de Tecnología Ambiental y Nanotecnología de la Universidad Tecnológica Fidel Velázquez.

" Docente investigador de la Unidad Académica de Estudios del Desarrollo de la Universidad Autónoma de Zacatecas. 
Capital's absorption of scientific knowledge. Considerations on the development of nanotechnologies

Absrtract. The historical dynamic of accumulation that has become integral to world economies has made possible the absorption of science and technology by capital, and nanotechnologies have not escaped that reality. Knowledge is being reappraised as an area of interest, and the repercussions could result in an increase of social inequality around the globe. In this article, the authors reflect on the implications of this absorption and what it means for nanotechnologies. The aim is to foster deeper discussion, from a critical perspective, on the development of this revolutionary technology package.

Keywords: absorption of science, knowledge, nanotechnologies, research, development. 


\section{Introducción}

El protagonismo de la ciencia y tecnología (СуT) en la acumulación de capital se ha incrementado como resultado de la profundización en la división del trabajo intelectual. La importancia social de la CyT se evalúa actualmente a partir de su inserción a los ciclos de valorización. Se consideran varios indicadores para develar su «pertinencia social», ya sea mediante artículos publicados en revistas de la corriente principal, patentes, servicio a la empresa o impacto en la productividad. Esto ha dotado a la CyT de un sentido instrumental y mercantil que, además, subordina el principio de servicio social de la ciencia al de generación de ganancia. Las nanotecnologías (NT) no escapan a esa realidad histórica, por lo que en este artículo se elabora una reflexión crítica a tal dinámica. Para ello desarrollamos cuatro secciones: en la primera se problematizan los conceptos de subsunción real y formal del trabajo; en la segunda se lleva a cabo un análisis equivalente con el paradigma del conocimiento; en la tercera se describen las características generales de las NT; y en la cuarta se sopesan las implicaciones de su subsunción a la acumulación del capital.

\section{Subsunción real de la cyT}

La base que da vida al sistema de producción mercantil y capitalista es la captura del plusvalor mediante el proceso de trabajo. En la actualidad la labor científica y tecnológica se ha subordinado al interés del capital en la búsqueda de nuevas fronteras de valorización. No es objetivo de este estudio efectuar una revisión acabada de las dinámicas de la CyT en el capital, 
sino reflexionar acerca de las implicaciones de la subsunción del conocimiento científico a la acumulación, en este caso de la mano de las NT.

A partir del concepto de subsunción real del trabajo que Marx define en El capital se desarrolla el análisis; para efectos de la presente investigación es preciso distinguir aquel del de subsunción formal del trabajo. Ambas nociones aleccionan respecto a la eterna misión del capital por controlar el proceso de trabajo, aunque varían en cuanto a su alcance sobre la base productiva y social. La subsunción real del trabajo involucra la creación de plusvalía en el proceso de valorización, un esquema «general» dentro del proceso productivo. En palabras de Marx:

El proceso de trabajo se convierte en el instrumento del proceso de valorización, del proceso de autovalorización del capital: de la creación de plusvalía. El proceso de trabajo se subsume en el capital (es su propio proceso) y el capitalista se ubica en él como dirigente (...) Es esto a lo que denomino subsunción formal del trabajo en el capital. Es la forma general de todo proceso capitalista de producción, pero es a la vez una forma particular respecto del modo de producción específicamente capitalista (Marx, 2009:54).

Todo proceso de trabajo genera valores de uso; en el capitalismo la función de éste es la creación de plusvalor, lo cual supedita la existencia de las fuerzas productivas a dicho objetivo (Marx, 2009). La subsunción real del trabajo implica la expansión máxima de la relación social del capital y, en consecuencia, la inserción de todas las actividades cotidianas hacia y para la acumulación. Ello incluye, desde luego, los avances de la ciencia, cuya mera subsistencia la controla el capital en beneficio de la clase dominante. Como sostiene Marx: 


\section{SUBSUNCIÓN DEL CONOCIMIENTO CIENTÍFICO AL CAPITAL}

Las fuerzas productivas sociales del trabajo, o las fuerzas productivas del trabajo directamente social, socializado (colectivizado) merced a la cooperación, a la división del trabajo dentro del taller, a la aplicación de la maquinaria y en general a la transformación del proceso productivo en aplicación consciente de las ciencias naturales, mecánica, química y de la tecnología, etcétera, con determinados objetivos, así como los trabajos en gran escala correspondientes a todo esto (...) Este desarrollo de la fuerza de trabajo objetivado, por oposición a la actividad laboral más o menos aislada de los individuos dispersos, etcétera, y con él la aplicación de la ciencia — ese producto general del desarrollo social — al proceso inmediato de producción: todo ello se presenta como fuerza productiva del capital, no como fuerza productiva del trabajo (...) La mistificación implícita en la relación capitalista en general, se desarrolla ahora mucho más de lo que se había y se hubiera podido desarrollar en el caso de la subsunción puramente formal (Marx, 2009:58-59).

Hoy el desarrollo de la CyT se integra a la socioeconomía del capital en forma de fuerza social productiva. El contexto histórico actual permite consolidar ese canal de acumulación, puesto que la integración de economías y mercados es un catalizador de dicha dinámica.

En el análisis de Guillermo Foladori (2016) relativo a la ciencia y su subordinación a la acumulación capitalista se alude a que el trabajo científico adquiere dos facetas. La ciencia, según el autor, se incorpora «como esfera de valorización del capital» cuando germina desde el seno de los departamentos de investigación y desarrollo (IyD) de las empresas, con la fi-

nalidad de incrementar la ganancia. A la vez, continúa Foladori, la ciencia adopta la forma de servicio hacia la sociedad mediante el financiamiento del Estado, así como los laboratorios y centros de investigación públicos. 
Aunque la búsqueda de la ganancia no sea un objetivo directo en ese esquema, también colabora en el proceso general de acumulación. El control sobre el conocimiento científico es una estrategia moderna para viabilizar el capital. Lo anterior se observa en la producción científica, la generación de nuevas aplicaciones técnicas y la construcción de nuevo conocimiento; con todo, las desigualdades sociales siguen incrementándose en el planeta. Por tanto, la raíz del problema no es la insuficiencia tecnológica o la falta de conocimiento científico; se ubica en el centro del sistema de producción, basado en la competencia y la ganancia, que si bien genera ganadores concibe mucho más perdedores. La subordinación o subsunción de la CyT, pasando por el conocimiento, termina consolidando una de las tantas contradicciones del modo de producción hegemónico. Es así que la subsunción real del trabajo científico solidifica un nuevo modo de valorización de capital.

\section{El conocimiento científico como esfera de valorización de capital}

El análisis del significado del conocimiento ha ido acompañado del debate sobre la noción de poder que sin duda trae consigo la adquisición del saber. En este caso el conocimiento se realiza socialmente mediante el proceso de subsunción real del trabajo científico.

Desde el empirismo baconiano («el conocimiento en su poder») y el estudio del uso político del conocimiento de Thomas Hobbes, hasta las obras detalladas de Michel Foucault, el debate sobre el tema ha sido álgido. El uso de conocimiento para definir la verdad socialmente aceptada y, 
con ello, fortalecer el control de poder, ha estado presente en el examen sobre el papel que el saber representa en todo grupo social, en el sentido de que el binomio verdad/poder beneficia a algunos al mismo tiempo que margina a otros.

La relación conocimiento/poder, presente a lo largo de la historia humana, pero amplificada por la potestad del capital, ha adquirido un nuevo vigor gracias a la revolución que han traído consigo las nuevas tecnologías, es decir, el impulso tecnocientífico que caracteriza al mundo moderno. En ese sentido, es importante profundizar sobre cómo el conocimiento, enmarcado en una era global, entra en una lógica de subsunción al capital, mercantilista, productivista, sin orientación humana y social.

Manuel Castells explica la forma en que la nueva revolución tecnológica significa la socialización de la información y del conocimiento en una lógica de objetivación de la innovación:

Lo que caracteriza a la revolución tecnológica actual no es el carácter central del conocimiento y la información, sino la aplicación de ese conocimiento e información a aparatos de generación de conocimiento y procesamiento de información/comunicación, en un círculo de retroalimentación acumulativo entre la innovación y sus usos (1997:61).

Según el autor, somos testigos de un nuevo paradigma civilizatorio, donde los medios de producción se estructuran sobre el conocimiento y se deja de lado la mano de obra y la materia prima. En este nuevo orden social, afirma, hay un desplazamiento del trabajo humano hacia actividades cada vez más especializadas, complejas, sofisticadas y con mayor vínculo con las innovaciones tecnocientíficas. El conocimiento, por consiguiente, 
conduce a los individuos hacia la especialización en ciertas ramas del saber, lo que limita en muchas ocasiones su conocimiento respecto al proceso de trabajo científico en general o acerca de las conexiones con otras ramas de la ciencia. Ello implica una parcialización y descontextualización del conocimiento, que orilla a los «hombres de ciencia» a trabajar en irrelevancias, en cuanto al beneficio de las clases sociales mayoritarias.

Asimismo, Castells asegura que el conocimiento está sustituyendo a los factores clásicos de la producción, ya que la producción de valor y riqueza ahora se miden en función del conocimiento adquirido, y no en la generación de bienes materiales o en la acumulación de trabajo en un objeto materializado. Esto parangona a lo que Foladori (2016) denomina como ciencia ficticia, al configurarse una forma de valorización de capital frente a bienes intangibles como aquellas patentes que no se traducen en proceso productivo. En suma, se atestigua una nueva era donde los factores tradicionales de producción y de generación de riqueza y plusvalor seguirán presentes, pero potencializados por el conocimiento científico al servicio del capital.

Ese fenómeno ha generado nociones como la de sociedad de la información o sociedad de conocimiento (knowledge society), ${ }^{1}$ las cuales denotan

${ }^{1}$ La diferenciación entre estos dos conceptos es importante. Para Abdul Waheed Khan, subdirector general de la Organización de las Naciones Unidas para la Educación, la Ciencia y la Cultura (UNESCO) para la Comunicación y la Información: «La sociedad de la información es la piedra angular de las sociedades del conocimiento. Mientras que yo veo el concepto de «sociedad de la información〉 como vinculado a la idea de la sinnovación tecnológicas, el concepto de ssociedades del conocimiento incluye una dimensión de transformación social, cultural, económica, política e institucional, y una perspectiva más pluralista y de desarrollo. En mi opinión, el concepto de ssociedades del conocimientos es preferible al de ssociedad de la información, ya que capta mejor la complejidad y el dinamismo de los cambios que están ocurriendo (...) El conocimiento en cuestión no sólo es importante para el crecimiento económico, sino también para empoderar y desarrollar todos los sectores de la sociedad». 
el uso económico que las sociedades modernas hacen del saber. Es decir, el conocimiento, el saber científico, tiene un valor de cambio en la generación de excedente, y se configura como una mercancía concreta en el mercado global. Con esta noción se evidencia la penetración del poder en la esfera tecnocientífica, lo que genera una nueva economía del conocimiento científico o, en términos de Raúl Delgado, un incipiente sistema imperial de innovación (Delgado, 2016). ${ }^{2}$

Frente a dicho antecedente, la socialización de la CyT no es un proceso neutro, imparcial y mucho menos equitativo, ya que se encuentra socialmente condicionado y responde, en última instancia, a esquemas propios de la valorización (Katz, 1994). En la sociedad del conocimiento se ha dotado a la ciencia de un valor comercial, donde la información adquiere un papel central en la reproducción de un esquema basado en la competencia. Semejante lógica ha alcanzado a las universidades, a los centros de investigación, a los laboratorios, a los cubículos y al espíritu de la ciencia en su conjunto, ello es perceptible en los indicadores de «productividad» a los que la labor científica se subordina. Se aprecia un fenómeno que trasciende la «desmaterialización» del mundo; nos dirige a una «hiperindustrialización» al mercantilizarse el conocimiento en forma de información intercambiable y codificable, convirtiéndose en el «recurso esencial» del capital (Drucker, 1998).

Para un análisis a mayor profundidad puede consultarse: http://www.ub.edu/prometheus21/ articulos/obsciberprome/socinfsoccon.pdf

${ }^{2}$ El estudio realizado por Raúl Delgado Wise sobre el «ecosistema» de Silicon Valley arrojó un análisis relativo a la "circulación de cerebros» en tanto fenómeno donde las grandes corporaciones, entre ellas la Administración Nacional de la Aeronáutica y del Espacio (NASA por sus siglas en inglés) y el Pentágono, generan grandes contratos con científicos, investigadores y emprendedores para adquirir sus patentes bajo el estandarte de que todos ganan, sin que ello, sostiene Delgado, sea así. 
Desde esa perspectiva, las nociones de valor adquieren nuevas dimensiones y fronteras. Estudios del Banco Mundial (2003; 2011; 2012) afirman que la diferencia real entre los países ricos y los pobres ya no puede medirse mediante el ingreso per capita o por el acceso y uso de sus recursos naturales, sino a partir del acceso y generación de conocimiento útil; saberes tecnificados, orientados a un fin y sujetos a la comercialización.

En su estudio Hacia las sociedades del conocimiento, la UNESCO (2005) destaca que los 29 países que concentran 80 por ciento de la riqueza mundial deben su bienestar en 67 por ciento al capital intangible (educación, investigación, ciencia y tecnología), 17 por ciento a sus recursos naturales y 16 por ciento a sus equipos. Estos datos aluden a que se otorga una preferencia a los esquemas de acumulación basados en la noción de sociedad del conocimiento, lo cual se ha convertido en marco de referencia para la mayoría de los países de la Organización para la Cooperación y el Desarrollo Económicos (OCDE) (el club de los países ricos) y para muchas naciones de economías emergentes y en desarrollo, especialmente de Asia Oriental y Sudoriental, América Latina y el Caribe, África Subsahariana, Europa Central y Oriental y la región de los Estados árabes (UNESCO, 2005).

Por su parte, en 2005 la Organización de las Naciones Unidas (ONU) convocó a la Cumbre Mundial de la Sociedad de la Información (CMSI) con el objetivo de reunir a quienes debatían al respecto. La discusión se centró en dos posiciones contrastantes: el instrumentalismo (vinculado al capital) y la necesidad de contextualizar socialmente el análisis.

El primer enfoque concibe a la sociedad de la información como un nuevo paradigma que asigna a la tecnología un papel causal en el ordenamiento social, ubicándola incluso como el motor del desarrollo global 
moderno. ${ }^{3}$ Es notoria una perspectiva instrumentalista del conocimiento, pero subordinada. Así, el instrumentalismo del conocimiento científico es uno de los avatares de la subsunción de la CyT al capital. Además, esta postura asevera que aquellos países que no logren incorporarse al tren del progreso y a los beneficios que trae consigo, quedarán fuera de la nueva redistribución. Es un planteamiento que coloca en desventaja a aquellos países que no invierten mucho en CyT, pues deja de lado el contexto socioeconómico e histórico que permite o impide el desarrollo científico y tecnológico de una nación; soslaya las condiciones objetivas que acompañan a la innovación, la concreción de proyectos de investigación y, en todo caso, el avance en la vinculación entre ciencia y necesidades sociales reales. Respecto al segundo enfoque, cabe resaltar que confrontó al primero durante el proceso de la Cumbre al confirmar que «en la nueva realidad que ha surgido, el nivel de desarrollo no se expresa tanto en el impacto que en él tienen las nuevas tecnologías, sino que el centro de análisis debiera desplazarse cada vez más hacia el impacto que tienen los contenidos involucrados en los procesos tecnológicos» (Sescovich, 2009:14). Por lo anterior, sostiene Sescovich que el uso y acceso a la información en las sociedades contemporáneas determina cómo es su gestión, tomando en cuenta las demandas específicas del desarrollo socioeconómico de la sociedad.

En consecuencia, la tecnología juega un papel fundamental al fortalecer el dominio y explotación de unos cuantos; la ciencia no es neutra, ni su rumbo es inexorable, porque normalmente responde a diversos intereses

${ }^{3}$ Bajo este concepto hubo quienes incluso sostuvieron que la sociedad de la información estaría suscrita al ámbito digital: lo que no estuviera digitalizado no formaría parte del corpus de información de la ciencia moderna (véase http://www.unesco.org/new/es/commu nication-and-information/resources/multimedia/photo-galleries/world-summit-on-theinformation-society-wsis/). 
hegemónicos. Se propone, en contraste, que la cadena de transmisión del beneficio científico sea de carácter humanista: las políticas públicas en ciencia y tecnología deben estar centradas en las necesidades de los seres humanos, enmarcadas dentro de los derechos sociales y de justicia distributiva, más allá de las estructuras y los intereses dominantes. Por ende, la acumulación de capital es el universo donde se desarrolla el conocimiento y en ese marco la institucionalidad global ha sido un elemento crucial para viabilizar su alcance. Agregamos a esta reflexión el impacto que tuvo la puesta en marcha del Consenso de Washington (CW) a finales de la década de los $1980 .{ }^{4}$

El CW puede caracterizarse como un conjunto de reformas tendientes a expandir la función de las fuerzas del capital mediante medidas coercitivas de carácter político-económico. Lo anterior implica orientar a los gobiernos de países en desarrollo y a los organismos financieros internacionales - Fondo Monetario Internacional (FMI), Banco Mundial (BM) y Banco Interamericano de Desarrollo (BID) — a evaluar avances en materia económica según indicadores financieros (Casilda, 2004). No es este el espacio para discutir la evolución del CW ni cada una de las reformas que impuso; sin embargo, basta con manifestar que el CW constató que la globalización genera sus propios instrumentos de regulación para que las relaciones de desequilibrio y desigualdad se mantengan, en beneficio de unos y en perjuicio de otros. Uno de esos instrumentos de regulación, que a la

${ }^{4}$ Consenso de Washington fue acuñado por John Williamson, quien también orquestó las diez medidas que los países no desarrollados debían adoptar si deseaban acceder a los préstamos de estos organismos y a las prebendas que le acompañan. Las medidas fueron: disciplina presupuestaria, cambio de prioridades en el gasto público, reforma fiscal, control en el tipo de interés, control del tipo de cambio, liberalización comercial, apertura a la Inversión Extranjera Directa (IED), adopción de políticas privatizadoras y desreguladoras, y adopción de un régimen de derechos de propiedad (Casilda, 2004). 
fecha se ha convertido en un pilar de la era global, es la manera en la que se adquiere, se usa y se socializa el conocimiento.

No obstante, existen varios criterios socioeconómicos que el CW dejó fuera para medir el «desarrollo»: educación, pobreza, cuidado y preservación del medio ambiente, equidad de género, entre otros. En cambio, colocó a la incorporación de los países no desarrollados como indicador de la «transformación productiva» y como objetivo del desarrollo mismo. ${ }^{5}$

El papel que jugaría el desarrollo científico y tecnológico de los países en desarrollo se supeditaría a los criterios que desde los países dominantes se dictaran. Cada uno de esos países debía adaptar sus planes de IyD e innovación a los criterios de medición de progreso y desarrollo, amén de la pérdida de apoyos económicos y financieros y penalizaciones severas por parte del BM o del FMI. ${ }^{6}$

De esa manera, el CW sentó la base de ideologización económica para América Latina, al convertir las políticas económicas en instrumentos de control para aquellos países interesados en ser partícipes del juego económico y financiero que la globalización traería consigo en busca de la aprobación de los organismos financieros internacionales.

Por tanto, el sistema tecnocientífico a escala global se configuró en ciertas regiones del mundo para servir como insumo de investigaciones, de «circulación de cerebros» de apropiación de patentes. Los territorios

${ }^{5}$ Se comenzó a hablar, inclusive, de un «fatalismo ambiental» como característica de los países latinoamericanos, donde la inequidad tendría una relación directa con la riqueza ecológica de la región, ya que el deterioro de las opciones de desarrollo, por ese hecho, sería más grande (Casilda, 2004:26).

${ }^{6}$ Hay diversos estudios por nación que demuestran los efectos de la implementación de dichas medidas, con resultados devastadores en las economías locales y en el desarrollo de una esfera científica nacional: Argentina (Batista, 2009); Uruguay (Casa, 2009); Colombia (Castro, 2001); países andinos (Moncayo, 2003); México (Martínez y Soto, 2012); entre otros. 
de las denominadas vanguardias científicas modernas ejemplifican el escenario, una de éstas son las NT.

\section{El mundo de las NT}

Las NT se definen como el campo de las ciencias dedicadas tanto al control como a la manipulación de la materia en la escala nanométrica. ${ }^{7}$ A esta escala los materiales no se comportan de la misma forma que los análogos en escala mayor (bulk). Algunos modifican sus propiedades: resistencia, absorción de luz, conductividad calórica y eléctrica, magnetismo, elasticidad y toxicidad.

Cabe destacar que la manipulación de la materia en dicha escala es un logro técnico que coloca a las NT en la vanguardia del conocimiento científico moderno (Acharya et al., 2011). Sin embargo, no se puede considerar una revolución tecnológica unilineal ni mucho menos homogénea. A la par del desarrollo de avances nanotecnológicos hasta hace poco inimaginables, se debate acerca del impacto en la salud humana y el medio ambiente, así como de los efectos en los ámbitos laborales y socioeconómicos. Los foros sobre avances en la materia han atestiguado la apertura a mesas donde se analizan desde asuntos de carácter ético/legal hasta implicaciones sociológicas del arribo de las NT a la vida del hombre.

Por tanto, la llegada de las NT marca el advenimiento de un sistema tecnológico convergente donde varias disciplinas y técnicas se integran (Roco y Bainbridge, 2003). Las NT son el núcleo de las cifras de inversión en IyD

${ }^{7}$ La escala nanométrica se encuentra en aquel espacio menor a un nanómetro (la millonésima parte de un milímetro, 10-9). A su vez, 1000 nanómetros equivalen a una micra (Serena, 2013). 
alrededor del mundo. En el nivel internacional, Estados Unidos, China, Alemania, Corea y Japón encabezan la lista. Estos países se caracterizan por desarrollar iniciativas específicas en IyD en torno a esa área; los estadounidenses destinan cerca de 2 mil millones de dólares anuales a financiar proyectos nanotecnológicos, mientras que la marca coreana Samsung destina más de 500 personas al desarrollo de investigaciones enfocadas en las NT (Gutiérrez, 2015). El volumen de mercado a escala mundial de las NT alcanzó, según estimaciones de Lux Research, el trillón de dólares en 2015; cifra que se disparará, de acuerdo con las tendencias de los últimos cinco años, hasta 4.4 trillones de dólares en 2018 (Lux Research, 2014). Se calcula que en 2015 en el nivel internacional las NT requirieron dos millones de trabajadores especializados, 50 por ciento en Estados Unidos y 25 por ciento en Europa (Gutiérrez, 2015), lo que indica una tendencia clara sobre la monopolización del mercado laboral y, por tanto, un avance nanotecnológico orientado hacia los intereses y necesidades de la industria de ese país.

Brasil, Argentina y México concentran 92 por ciento de la inversión que se hace en América Latina para las NT: Brasil invierte 1.2 por ciento de su PIB, Argentina 0.6 por ciento y México 0.4 por ciento, con una cifra próxima a 44 mil millones de dólares en 2014 (Small, 13 de mayo de 2014). Foladori e Invernizzi reportan, con base en Woodrow Wilson International Center for Scholars, la existencia en el mercado de mil 317 productos habilitados por las NT en 2011 (Foladori e Invernizzi, 2012).

Las estructuras nano han existido desde tiempos memorables: poseen nanopartículas las emisiones volcánicas; también se encuentran en las partículas generadas por los motores a combustión. Estudios recientes han comprobado que las legendarias espadas de Damasco, famosas por su fortaleza durante la Edad Media, debían su fuerza a nanotubos de carbono en 
su estructura (El Mundo, 16 de noviembre de 2006). En las copas de Lycurgus, al igual que en algunos vitrales de los edificios europeos de la época medieval, el efecto refractivo era por la combinación de nanopartículas de oro y plata en su composición (Freestone et al., 2007). Asimismo, estudios con difracción de rayos X han descompuesto el «azul» en las ruinas mayas, un compuesto que ha permanecido constante a pesar del tiempo y las condiciones atmosféricas. Los estudios demuestran la presencia de arcilla palygorskita y añil (Chiari et al., 2008). Pero es hasta 2001 que, de la mano del capital estadounidense, chino y europeo, esas estructuras se están manipulando y mercantilizando a escala global.

No obstante, las aplicaciones nanotecnológicas en las esferas de la vida las han colocado en la mira de diversas ramas industriales y de grandes corporaciones multinacionales. Primero, como plataforma de una revolución que mejoraría los productos y, segundo, como un paquete de herramientas que incentivará su competitividad comercial. Las NT no se enfocan en una rama específica del conocimiento, son multiseccionales:

A diferencia de las revoluciones tecnológicas anteriores, donde el cambio estaba centrado en la fuente de energía, en la maquinaria o en determinados sectores económicos, aquí estamos frente a una revolución tecnológica que no es sectorial, y que por el grado de integración del mercado mundial de hoy día tendrá un impacto prácticamente simultáneo en todo el mundo (Foladori, 2009:144).

Sus aplicaciones son innumerables: implementación de nanobiosensores capaces de detectar microorganismos patógenos en los alimentos, a fin de evitar el consumo de comida caduca; incorporación de nanoestructuras 
especializadas que eficientan las placas solares para la reducción del consumo energético; desarrollo de nanocatalizadores que podrían conseguir reacciones químicas útiles en el tratamiento y saneamiento de agua. La industria farmacéutica y de cosméticos invierte cantidades millonarias en este ámbito, pese a las constantes críticas por la falta de estudios toxicológicos que en el mercado ya existen nanofabricados (European Commission, 2013).

Complementariamente, su uso ha permeado en diversos contextos industriales: sostenibilidad del medio ambiente, salud humana, aplicaciones militares, búsqueda de nuevas fuentes de energía, desarrollo de nuevos materiales, medicina, entre otros. Sin embargo, esta serie de aplicaciones ha transitado de manera inmediata de un escenario de promesas y grandes expectativas en las décadas de 1980 y 1990, a otro donde lo que impera son las incertidumbres por los riesgos, aún desconocidos, y las potenciales implicaciones sociales. Si bien se visualizan los beneficios técnicos del conocimiento nanocientífico, también se analizan las implicaciones sociales. ${ }^{8}$

Toda implementación de nuevas tecnologías trae consigo escenarios caracterizados por las contradicciones. En ese sentido, las corporaciones y los grandes capitales se encuentran ante una paradoja. No hay protocolos de evaluación toxicológica para los nanomateriales antes de que logren introducirse al mercado. Las posturas apologéticas aducen que no introducen elementos nuevos en la naturaleza, sólo los integran en distinta escala.

${ }^{8}$ La Organización Mundial de la Salud (OMS) asegura que anualmente en el mundo el uso de sustancias químicas mata a cerca de 4.9 millones de personas e incapacita a 85 millones más. El llamado de atención se enfoca entonces en la regulación sobre el uso de productos químicos en cualquier producto consumido/utilizado por los seres humanos y en este grupo también se encuentran los nanomateriales (Foladori e Invernizzi, 2005; Bowman y Hodge, 2007; García, 2013; Azoulay, Foladori y Jensen, 2013). 
En efecto, es el tamaño lo que produce características físicas y químicas desconocidas. El gran negocio alrededor de las NT gira en torno de este procedimiento, cuyo requisito ineludible es el desarrollo de nuevos productos y procedimientos, aunque aquí se limite, en infinidad de ocasiones, al tamaño. ${ }^{9}$ Los estudios sobre los riesgos de los nanomateriales manufacturados han incrementado; en PubMed (2005) se ubicaron 24 artículos y en 2015 cerca de 4 mil (Shvedova, Pietroiusti y Kagan, 2016).

Hasta el momento no existen estudios suficientes respecto a la manera en que los nanomateriales afectarán la cadena trófica a largo plazo, ¿cuál será su impacto en la biósfera?, ¿cómo alterarán a los ecosistemas, a los diferentes hábitats y a la biodiversidad en general? En realidad, no lo sabemos. Incluso, se empieza a discutir en torno de las implicaciones del manejo de la nanobasura y sobre el tipo de residuos que se generarán (Song et al., 2009); además se examina si el sistema biológico es capaz de asimilar materiales con los que no ha evolucionado desde hace millones de años. Los riesgos al medio ambiente se vinculan de forma directa con la toxicidad de las nanopartículas (RS y RAE, 2004; Liou et al., 2012)..10 Se discute la posibilidad de desarrollar nanotecnología verde (Sifontes, 2014). Otros temas sobresalientes también

${ }^{9}$ La lógica mercantilista tras la patente responde a los cuasi monopolios que una innovación puede otorgar. La patente se reserva el derecho de «invención» por un determinado número de años (generalmente veinte), de ahí que los productos nuevos sean por lo común costosos, ya que la producción protegida bajo patente garantiza que otros productos en el mercado no estén cubiertos con esa patente, lo que asegura el cuasi monopolio u oligopolios. Los oligopolios garantizan una tasa de ganancia elevada, en especial desde que varias firmas se asocian para minimizar la competencia de precios (Wallerstein, 2006).

${ }^{10}$ Un cuestionamiento severo al conocimiento nanotecnológico lo hace la Organización de las Naciones Unidas (ONU), que asegura que de las aproximadamente 144 mil sustancias químicas utilizadas por diversas industrias relacionadas con la nanotecnología, sólo 8 mil (5.5 por ciento del total) han sido caracterizadas, y que de ese número sólo 600 han sido prohibidas — varias de ellas utilizadas para la fabricación de nanopartículas (UN-Water, 2013). 
se han excluido del desarrollo de las NT: el papel que desempeñan en tanto herramienta científica para resolver problemas humanos, como la vejez, la longevidad, el combate a enfermedades y las prótesis; las repercusiones en el ámbito laboral (Fatkhutdinova et al., 2016); y los impactos en la esencia humana (García, 2013).

\section{Reflexión sobre la subsunción del conocimiento nanotecnológico}

La CyT, el conocimiento, el saber y la IyD se subordinan a los procesos de valorización del capital, es una realidad histórica que no pueden eludir las NT. En otras palabras, las fuerzas que impulsan se fundamentan en las leyes de acumulación y en la reproducción de un sistema de relaciones sociales históricamente determinadas. Dicho sistema ha encontrado en el conocimiento científico un aliado para perpetuar relaciones donde las bonanzas no se redistribuyen equitativamente. El poder de las NT es centralizado y podría ampliar la diferenciación económica entre individuos, grupos, regiones, naciones y clases sociales.

De acuerdo con Juan Pérez Ventura (2016), no se puede hablar sólo de la confrontación entre el Norte y el Sur, donde el Norte está conformado por las potencias tradicionales y el Sur por las economías en constante crisis y países subdesarrollados altamente dependientes. La geoeconomía divergente ha ampliado sus fronteras y reivindica la reflexión sobre cómo la esfera del conocimiento participa en el contraste social. Al tomar como referencia el Producto Interno Bruto (PIB) per cápita de la nación más adelantada del mundo, Estados Unidos, y al compararlo con diferentes 
regiones a lo largo del tiempo, nos percatamos que la desigualdad se ha incrementado desde la década de los 1960: 206 por ciento para América Latina, 196 por ciento para el Sudeste Asiático y 207 por ciento para África Subsahariana (Hickel, 2016).

Sostenemos la hipótesis de que las NT no son ajenas a las estructuras socioeconómicas globales y que son asimiladas a un esquema desigual en cuanto acceso al conocimiento y al beneficio de su oferta tecnocientífica. Ello se ilustra al analizar la trayectoria de la cadena de valor, en la que los países industrializados invierten grandes sumas en IyD, patentan los resultados y guían sus investigaciones en función del segmento de mercado que genere mayor beneficio. Así, es poco probable que aun cuando sectores importantes de la población viven en condiciones que van de pobreza a extrema pobreza, se asegure que las NT contribuyen a reducir las brechas de desigualdad e inequidad imperantes en la actualidad (Záyago y Foladori, 2010).

Los países y las regiones con escaso desarrollo industrial no logran alcanzar los beneficios que trae consigo la comercialización de las NT. Según un estudio sobre patentado nanotecnológico de la OCDE, Estados Unidos, China, Corea del Sur, Canadá y Alemania lideraban el número de aplicaciones de patentes, mientras que los países en vías de desarrollo no formaban parte de los primeros diez lugares (Dang et al., 2010). Las NT aparecen como una obligación y no como una opción. México, al igual que América Latina, se ha embarcado en esa «receta para el desarrollo» basada en tales tecnologías para competir en el mercado (Záyago y Foladori, 2010). Son varias las investigaciones que advierten del impacto social que las NT podrían traer consigo en tanto que se desplieguen en un marco de relaciones sociales inequitativas (Cheang, 2006; Barrere, 2008; Sandler, 2009; Záyago y Foladori, 2010; Foladori e Invernizzi, 2012). Además, los costos alrededor 
de la IyD de las NT, la trayectoria histórica en ciencia y la formación de personal calificado coloca a los países no desarrollados en franca desventaja ante los países industrializados (RS y RAE, 2004). Son las corporaciones multinacionales quienes sistémicamente poseen control absoluto sobre la producción, la distribución y la riqueza generada por el progreso nanotecnológico, sin que ello signifique una orientación hacia la satisfacción de las necesidades de las mayorías. Por ende, algunos sostienen que esta nueva tecnología conllevará a una mayor concentración de poder y riqueza económica (ETC Group, 2004; Cheang, 2006). Es evidente, por ejemplo, al ubicar a las primeras diez instituciones con más patentes nanotecnológicas hasta 2011, lo que arroja a empresas transnacionales o instituciones asentadas en grandes economías, en orden descendente: Samsung (Corea del Sur), IBM (Estados Unidos), Hon Hai (Taiwán), Universidad de California (Estados Unidos), Universidad Tsinghua (China), 3M (Estados Unidos), MIT (Estados Unidos), GenAsys (Australia), Xerox (Estados Unidos) y Dupont (Estados Unidos) (Kaiser y Moore, 2012). No hay empresas y corporaciones de países en vías de desarrollo en el top 30."

Históricamente, los resultados de las innovaciones tecnológicas sólo han beneficiado a minorías muy localizadas (Meridian Institute, 2005). De manera que desde la promesa en los 1950 de las bondades de la energía nuclear (la cual reduciría el costo de la generación de energía, al contemplar la posibilidad de satisfacer la demanda de un mayor número de personas), o la Revolución verde de la década de 1960 (que aseguraba la dotación de alimentos a la gente más necesitada y eliminaba de este modo las hambrunas en el mundo) y más recientemente los avances en biomedicina

${ }^{11}$ Las excepciones serían China e India que tienen mercados internos muy amplios, lo que hace viable una dinámica de producción y consumo por encima de la media internacional. 
e ingeniería genética de los 1990 (que prometían la impartición de salud y medicinas para la mayoría de la población mundial), las expectativas del beneficio social general no se lograron. La meta técnica del supuesto beneficio de las NT también podría diluirse al ser insertada en una plataforma social y económica promotora de la desigualdad (Foladori e Invernizzi, 2005). En adición, debe analizarse cómo las aplicaciones de las NT afectarán las ventajas productivas de países en vías de desarrollo a partir de la exportación de materias primas. ${ }^{12}$ Existen varios autores, organizaciones internacionales, sindicatos, asociaciones de consumidores que también apoyan esta crítica, en cuanto a que las NT harán viable la desigualdad social (Dayrit y Enríquez, 2002; ETC Group, 2004; RS y RAE, 2004; Meridian Institute, 2005; McGrail, 2010).

Si ya se hizo referencia a una brecha digital entre el Norte y el Sur global, es posible, de igual modo, hablar de la conformación de una brecha nanotecnológica Norte-Sur. La postura avanzada por SciDevNet se conoce como instrumental, aunque oculta su verdadero cariz como avatar del capital. Se basa en la idea de que la ciencia posee un carácter neutral, transferible a diferentes contextos sin problema alguno; una ciencia apolítica, sin historia, sin contexto, sin intereses y sin reivindicaciones de los grupos que la impulsan. Esto implica un examen contradictorio sobre lo que significa el avance de la ciencia general bajo la mano humana (Ruiz y Noguera, 2011). Los instrumentalistas enfatizan en la capacidad técnica que la ciencia ofrece para solucionar problemas sociales, sin cuestionar los

${ }^{12}$ Ambos autores coinciden que aunado a esto un elemento más que abrirá la brecha entre países desarrollados y los no desarrollados, como producto de la nanotecnología, será el proceso de monopolización de las patentes, la cual concentrará la riqueza en un puñado de corporaciones en perjuicio de las grandes mayorías (Foladori e Invernizzi, 2005). 
asuntos de carácter político, económico, social e incluso cultural presentes detrás del desarrollo tecnológico (Díaz, 1991; Katz, 1994; Resnik, 2012).

Por otra parte, se encuentra la posición contextual. En esa vertiente, la tecnología materializa las relaciones sociales, políticas, económicas de una nación o de un grupo social. El desarrollo tecnológico tiene una arista de dominación, que refleja las estructuras materiales subyacentes; no es independiente de un contexto que le determina y está socialmente condicionado: «Si en Latinoamérica el desarrollo de la nanotecnología se desconecta de las necesidades sociales e ignora las implicaciones sociales de su uso, es probable que coloque en una posición más vulnerable a quienes están ya marginalizados» (Invernizzi et al., 2007).

En dicha postura, la idea de que los productos nanotecnológicos ayudarán a reducir la brecha entre Norte y Sur es severamente rebatida. Un incremento en la productividad, la competitividad, la composición del PIB, las ventas tecnológicas, no necesariamente implica una reducción de la pobreza y la consecuente inequidad. Por ejemplo, en la edición 2015-2016 del Índice Global de Competitividad (IGC) del Foro Económico Mundial, México avanzó cuatro lugares al pasar del lugar 61 al 57 (Imco, 2016). En ese mismo periodo, según el Consejo Nacional para la Evaluación de la Política de Desarrollo Social, se incrementó en 2 millones de personas el número de pobres en el país (Coneval, 2016). Por tanto, un incremento en competitividad no se traduce mecánicamente en menos pobreza. Un planteamiento de esa naturaleza supone una rusticidad analítica o, al menos, una ingenuidad científica. No es vano que el desarrollo de las NT, guiado por las grandes corporaciones, tenga como centro de atención las necesidades de los grupos sociales con mayor poder adquisitivo y no el de las mayorías pobres (Invernizzi et al., 2007). Esto, sin embargo, no es un 
fenómeno nuevo en cuanto a los desarrollos tecnológicos. La industria farmacéutica, la química, la manufacturera y demás, han dejado testimonio de que el mercado objetivo es el que contiene mayor poder de compra y no aquel que implica mayor beneficio social. La satisfacción de necesidades a partir de bienes suntuarios mueve la lógica del capital y de la CyT en el mundo moderno.

\section{Conclusiones}

En la sociedad del conocimiento y de la información, el debate sobre el papel que cumple (y debería cumplir) el saber tecnocientífico ha adquirido una nueva faceta. La reflexión aquí vertida busca motivar el debate concerniente a un aspecto de la integración de la CyT a las dinámicas del capital. Hemos analizado cómo la subsunción real del trabajo explica las maneras modernas en las que el capital ha posicionado al conocimiento, dotándole de nuevas características e implicaciones.

Así, las sociedades contemporáneas observan el desarrollo de la CyT como un elemento sine qua non del progreso y a la innovación como el principal indicador del desarrollo técnico; el estadio de avance en el que se halla cualquier sociedad es medido en función de su participación —o no- en los circuitos de acumulación que el capitalismo moderno ha modelado.

Adicionalmente, una parte importante del debate en este punto se centra alrededor del impacto y la fuerza que esta noción de progreso posee en el ser humano; es decir, el proceso de individualización ha encontrado un ancla fundamental en la interiorización que los sujetos tienen sobre la 
idea del progreso (subjetividad), midiendo su éxito social e individual en su participación dentro del proceso de acumulación, ya sea como usuarios de los gadgets (fetichizados) o como parte del proceso de objetivación del capital (ahora vía CyT). Adicionalmente, en la sociedad del conocimiento, el proceso de socialización del capital ha identificado en las nociones de progreso social e individual un elemento crucial de diferenciación social. Las nuevas tecnologías han abierto de modo significativo la brecha entre aquellas naciones que tienen protagonismo en la acumulación y han ampliado los márgenes de estratificación y determinismo económico a escala global.

Un ejemplo de lo anterior es la función que en las sociedades modernas están desempeñando las NT. Desde su concepción generaron expectativas respecto al impacto que tendrían en diferentes áreas de la actividad humana, al grado que se pensó, erradamente, que solucionarían problemas propiciados por las estructuras del capital. El avance de las NT ha experimentado un desarrollo totalmente desigual, manifiesto en la notoria inclinación de las investigaciones en el ámbito internacional a responder a necesidades sociales propias de los países que mayores montos de inversión tienen en éstas: países del denominado primer mundo, con necesidades que poco tienen que ver con urgencias sociales vinculadas a pobreza, marginación o zonas marginadas. En coyunturas determinadas y espacios específicos las NT podrían coadyuvar a resolver ciertos problemas sociales, pero nunca solucionarán las causas socioeconómicas de origen. La CyT, así como el conocimiento, se han convertido en fronteras dinámicas en el proceso de valorización del capital. Se impulsan en función de su capacidad de mejorar las tasas de ganancia y no en función del beneficio social. Las NT no escapan a la trayectoria histórica y económica del capitalismo. Un importante sector científico cuestiona la manipulación de materiales en torno 
a los aspectos de toxicidad y riesgos que ocasionan. Existen debates cada vez más amplios acerca de los impactos que las NT pueden traer consigo tanto a la salud humana como al medio ambiente y la «nueva naturaleza».

Pese a ello, las NT siguen avanzando a pasos agigantados en las principales economías del mundo. Los montos de inversión, como hemos visto, alcanzan cifras sin precedentes. Los grandes consorcios corporativos y las principales transnacionales reclaman en la IyD de las NT la generación de ganancia extraordinaria, además los gobiernos más ricos del mundo destinan cuantiosas sumas económicas y de personal calificado al desarrollo de saber alrededor de las NT. El rezago en materia técnica es cada vez mayor y los procesos sociales son innumerables: fuga de cerebros, apropiación de los bienes intangibles (conocimiento, patentes), control monopólico del conocimiento nanocientífico, precarización y outsourcing de los procesos de innovación nanotecnológica, entre otros. Las recetas de crecimiento establecidas por organismos internacionales como la OCDE, el BM, el BID y la ONU parecen no tomar en cuenta los contextos científicos, subordinados al capital, donde éstas se desarrollan y promueven un escenario ideal para que las desigualdades se impongan.

\section{Referencias}

Acharya, Amitav, Kamilla Sushanta, Manasa Nayak y Gouri Sankar Roy (2011), "Nano the revolution of 2lth century», Latin-American Journal of Physics Education, 5(2), pp. 418-422, en https://dialnet.unirioja.es/servlet/autor? codigo $=2942182$ 
Azoulay, David, Guillermo Foladori y Rye Senjen (2014), Social and environmental implications of nanotechnology development in Asia-Pacific, Genova, IPEN Press Releases.

Batista, Johanna (2009), El condicionamiento al capital externo como instrumento para la implementación de reformas neoliberales en América Latina: la aplicación del Consenso de Washington en Argentina, Bogotá, Facultad de Relaciones Exteriores.

Bowman, Diana y Graeme Hodge (2007), «A small matter of regulation: an international review of nanotechnology regulation», Science and Technology Law Review, VIII, pp. 1-36, en http://stlr.org/volumes/volume-viii-2006-2007/ bowman/

Casa, Mauro (2009), «Análisis crítico del Consenso de Washington y de sus principales repercusiones para el caso uruguayo», en https://www.academia. edu/745434/an\%c3\%81lisis_cr\%c3\%8dtico_del_consenso_de_washington_y_ de_sus_principales_repercusiones_para_el_caso_uruguayo

Casilda, Ramón (2004), «América Latina y el Consenso de Washington», Boletín Económico de ICE (2803).

Castells, Manuel (1997), La era de la información: economía, sociedad y cultura. La sociedad red, volumen 1, Madrid, Alianza.

Castro, Robinson (2001), «Análisis crítico del Consenso de Washington», Revista Panorama Económico, 9(1), pp. 44-49.

Cheang, Juan Carlos (19-23 de junio de 2006), «Nanotecnología: ¿hacia dónde nos llevará?», ponencia presentada en el I Congreso Iberoamericano de Ciencia, Tecnología, Sociedad e Innovación, Ciudad de México.

Chiari, Giacomo, Roberto Giustetto y David Carson (2008), «Azul Maya: una maravillosa nanotecnología precolombina», Boletín de Monumentos Históri$\cos$ (12), pp. 39-48, en http://www.boletin-cnmh.inah.gob.mx/boletin/bole tines/3EV12P39.pdf 
Consejo Nacional para la Evaluación de la Política de Desarrollo Social (Coneval), Reporte de medición de pobreza 2015-2016, en http://www.coneval.org. $\mathrm{mx} /$ Medicion/Paginas/PobrezaInicio.aspx

Dang, Yang, Yulei Zhang, Li Fan, Hsinchun Chen y Mihail Roco (2010), «Trends in worldwide nanotechnology patent applications: 1991 to 2008», Journal of Nanoparticle Research, 12(3), pp.687-706, en https://www.ncbi.nlm.nih.gov/ pmc/articles/PMC2988206/

Dayrit, Frederich y Ernesto Enriquez (2002), «Nanotechnology issues for developing economies», en Greg Tegart (ed.), Nanotechnology: the technology for the 21st century, Bangkok, APEC Center for Technology Foresight.

Delgado Wise, Raúl (septiembre de 2016), «Reestructuración de los Sistemas Nacionales de Innovación bajo la égida neoliberal», Revista Pr (presentación PPT). Díaz, Carlos (1991), En el jardín del Edén, Salamanca, Esteban.

Drucker, Peter (1998), La sociedad poscapitalista, España, Norma.

El Mundo (16 de noviembre de 2016), «Los nanotubos de carbono, el secreto de las espadas de Damasco», El Mundo, en http://www.elmundo.es/elmundo /2006/11/15/ciencia/1163606031.html

ETC Group (2004), Communiqué (85), en www.etcgroup.com

European Commission (2013), «Nanotechnologies: principles, applications, implications and hands-on activities. European Union», en http://ec.europa. eu/research/industrial_technologies/pdf/nano-hands-on-activities_en.pdf Fatkhutdinova, Liliya M., Timur Khaliulliin, Olga Vasiliyeva y Anna Shvedova (2016), «Fibrosis biomarkers in workers exposed to MWCNTs», Toxicology and Applied Pharmacology, en doi: 10.1016/j.taap.2016.02.016

Foladori, Guillermo (2007), «Nanotecnología, salud y pobreza. ¿Cuáles son las expectativas?», en Juan Ramón Coca (coord.), Varia Biológica. Filosofía, Ciencia y Tecnología, León, Universidad de León, pp. 43-63. 


\section{SUBSUNCIÓN DEL CONOCIMIENTO CIENTÍFICO AL CAPITAL}

(2009), «La gobernanza de las nanotecnologías», Revista Sociológica, 24(71), pp. 125-153.

(2016), «Ciencia ficticia», Estudios Críticos del Desarrollo, IV, pp. 4l-66.

Foladori, Guillermo y Noela Invernizzi (2005), «Nanotecnología: ¿beneficios para todos o mayor desigualdad?», ponencia presentada en la Reunión Inaugural de la International Nanotechnology and Society Network, Universidad Estatal de Arizona.

(2012), Implicaciones sociales y ambientales del desarrollo de las nanotecnologías en América Latina y el Caribe, México, Red Latinoamericana de Nanotecnología y Sociedad.

Freestone, Ian, Nigel Meeks, Margaret Sax y Catherine Higgitt (2007), «The Lycurgus cup. A Roman nanotechnology», Gold Bulletin, 40(4), pp. 270-277, en http://master-mcn.u-strasbg.fr/wp-content/uploads/2015/09/lycurgus.pdf

García, Miguel y Guillermo Foladori (2013), «Divulgación de las implicaciones sociales y ambientales de las nanotecnologías», Revista Digital Universitaria, 14(4), en http://www.revista.unam.mx/ojs/index.php/rdu/article/view/162

Gutiérrez, Federico (2015), «Nanotecnología», Slide Share, en http://es.slside share.net/fede ricoggutierrez2/investigacion-sobre-nanotecnologia-2015

Hickel, Jason (2016), "Global inequality may be much worse than we think», The Guardian, en www.theguardian.com/global-development-professionals-net work/2016/apr/08/global-inequality-may-be-much -worse-than-we-think

Instituto Mexicano para la Competitividad (Imco) (2016), «México avanza cuatro lugares en la competitividad vía WEF», en http://imco.org.mx/competiti $\mathrm{vidad/mexico-avanza-cuatro-lugares-en-competitividad-via-wef/}$

Invernizzi, Noela, Guillermo Foladori y David Maclurcan (2007), «Nanotechnology's controversial role of the south», Science, Technology \& Society, 13(1), pp. 123-148. 
Katz, Claudio (1994), «Controversias sobre el cambio tecnológico», Revista Problemas del Desarrollo, 25(97), pp. 129-153.

Kaiser, Iona, Carey Jordan y Valerie Moore (2012), «Nanotechnology patent survey: who will be the leaders in the fifth technology revolution?», en https:// www.mwe.com/ /media/files/thought-leadership/publications/2012/10/ nanotechnology-patent-survey-who-will-be-the-lea__/files/nanotechnology_ patent_survey/fileattachment/nanotechnology_patent_survey.pdf

Liou, Saou et al. (2012), «Epidemiological study of health hazards among workers handling engineered nano-materials», Nanoparticle Research, 14(878).

Lux Research (2015), «Nanotechnology update: corporations up their spending as revenues for nano-enabled products increase», en https://members. luxresearchinc.com/research/report/13748

Martínez, Rubí y Ernesto Soto (2012), «El Consenso de Washington: la instauración de las políticas neoliberales en América Latina», Política y Cultura (37), pp. 35-64. Marx, Karl (2009), El capital, libro I, capítulo VI, México, Siglo XXI.

McGrail, Stephen (2010), «Nano dreams and nightmares: emerging technoscience and the framing and (re)interpreting of the future, present and past», Journal of Future Studies, 14(4), pp. 23-48.

Meridian Institute (2005), «Nanotechnology and the poor: opportunities and risks», en www.nanoandthepoor.org

Moncayo, Edgard (2003), «Resultados de las reformas del Consenso de Washington en los países andinos: estabilización incompleta, profundización de los desequilibrios sociales y crecimiento precario», Revista Economía y Desarrollo, 2(1), pp. 73-95.

Observatorio Iberoamericano de la Ciencia, la Tecnología y la Sociedad (2008), La nanotecnología en Iberoamérica. Situación actual y tendencias, Argentina, Organización de Estados Americanos. 
Pérez Ventura, Juan (2016), «El orden económico histórico a nivel global», en https://vaventura.com/divulgacion/economia/orden-economico-historico -nivel-global/

Resnik, Mario (2012), Paradigmas en ciencia politica, Buenos Aires, Eudeba.

Roco, Mihail y William Bainbridge (2003), Converging technologies for improving human performance. Nanotechnology, biotechnology, informatic technology and cognitive science, Netherlands, Kinner Academic Publishers.

Royal Society y The Royal Academy of Engineering (RS y RAE) (2004), Nanoscience and nanotechnology: opportunities and uncertainties, London, RS/RAE, en www.raeng.org.uk y www.royalsoc.ac.uk/policy

Ruiz, Rosaura y Ricardo Noguera (2011), «Sobre la neutralidad de la ciencia», El Universal, en http://archivo.eluniversal.com.mx/editoriales/52833.html Sandler, Ronald (2009), Nanotechnology: the social and ethics issues, New York, Woodrow Wilson International Center for Scholars.

Serena, Pedro (abril 2013), «Acercando la nanotecnología a la sociedad: la exposición «Un paseo para la humanidad»», Revista Digital Universitaria, 14(4), en http://www.revista.unam.mx/vol.14/num4/art29/

Sescovich, Sonia (2009), La gestión de las personas: un instrumento para humanizar el trabajo, Chile, Libros en Red.

Shvedova, Anna, Antonio Pietroiusti y Valerian Kagan (2016), «Nanotoxicology ten years later: lights and shadows», Toxicology and Applied Pharmacology, en http://dx.doiorg/10.1016/j.taap.2016.02.014

Sifontes, Angela (2014), «Biosíntesis de nanomateriales: hacia el avance de la nanotecnología verde», Revista Mundo Nano, 7(13), pp. 56-68, en http://www. revistas.unam.mx/index.php/nano/article/view/48709/43759

Small Carmona, Andrea (13 de mayo de 2014), «Tres países concentran 92\% de inversión en I+D en la región», SciDevNet, en http://www.scidev.net/america- 


\section{Domingo Rafael CAStañeda Olvera | Edgar Záyago lau}

latina/i-d/noticias/tres-pa-ses-concentran-92-de-inversi-n-en-i-d-en-laregi-n.html

Song, Yang, Xio Li y Xan Du (2009), «Exposure to nanoparticles is related to pleural effusion, pulmonary fibrosis and granuloma», European Respiratory Journal, 34(3), pp. 559-567.

Organización de las Naciones Unidas para la Educación, la Ciencia y la Cultura (UNESCO) (2005), Hacia las sociedades del conocimiento, París, UNESCO, en http://unes doc.unesco.org/images/0014/001419/141908s.pdf

UN-Water (2013), Managing water under uncertainty and risk, Washington, UN-Water, en www.unwater.org/documents.html

Waheed Khan, Abdul (21 de abril de 2005), «Sociedad de la información/Sociedad del conocimiento», en http://www.ub.edu/prometheus21/articulos/ obsciberprome/socinfsoccon.pdf

Wallerstein, Immanuel (2006), Análisis de sistemas-mundo. Una introducción, México, Siglo XXI.

World Bank (2003), «Aprendizaje permanente en la economía mundial del conocimiento: desafíos para los países en desarrollo», en http://documentos. bancomundial.org/curated/es/346561468281387271/Aprendizaje-permanenteen-la-economia-global-del-conocimiento-desafios-para-los-paises-endesarrollo

(2011), «Knowledge for development», en http://siteresources.worldbank. org/PROJECTS/Resources/40940-1316471060185/KnowledgeBookletcomplete. pdf

(2012), «Resultados para el desarrollo: el conocimiento al servicio de la prosperidad y la erradicación de la pobreza», en http://www.bancomundial. org/es/news/speech/2012/10/08/delivering-development-harnessing-know ledge-build-prosperity-end-poverty 
SUBSUNCIÓN DEL CONOCIMIENTO CIENTÍFICO AL CAPITAL

Záyago, Edgar y Guillermo Foladori (2010), «La nanotecnología en México: un desarrollo incierto», Economía, Sociedad y Territorio, 10(32), pp. 143-178. 